\title{
Grupos de pesquisa em cursos de Educação Física com pós-graduação "stricto sensu" no Brasil: análise temporal de 2000 a 2008
}

CDD. 20.ed. 016.796

796

\author{
Giovâni Firpo Del DUCA* \\ Leandro Martin Totaro GARCIA* \\ Kelly Samara da SILVA* \\ J uarez Vieira do NASCIMENTO*
}

*Universidade Federal

de Santa Catarina.

\section{Resumo}

0 objetivo da pesquisa foi descrever a evolução dos grupos de pesquisa em cursos de Educação Física com programas de pós-graduação "stricto sensu" no Brasil e sua produção intelectual no período de 2000 a 2008. Trata-se de um estudo descritivo, incluindo análise secundária de dados conduzida em 2010 com informações do Diretório dos Grupos de Pesquisa no Brasil, do Conselho Nacional de Desenvolvimento Científico e Tecnológico. Analisou-se grupos de pesquisa; razões de pesquisadores e de estudantes por grupos de pesquisa; produção de artigos nacionais, artigos internacionais, livros e capitulos de livros, conforme macrorregiões geográficas; anos de coleta; número de cursos em Educação Física, pesquisadores e estudantes. As razões, frequências absolutas e relativas foram utilizadas na análise estatística descritiva dos dados. Os primeiros grupos de pesquisa em Educação Física no Brasil foram criados na década de 80, e em 2008 totalizaram 387. Observou-se diminuição da representatividade de grupos criados em instituições com programas "stricto sensu" (até 2000: 75,6\%; de 2005-2008: 43,9\%). No ano 2000, havia 453 pesquisadores e 479 estudantes de cursos de Educação Física. Identificou-se aumento nos anos 2002, 2004, 2006 e 2008, em comparação ao ano anterior (pesquisadores: aumento de $89 \%$, 71\%, 34\% e 27\%; estudantes: $83 \%$, 76\%, 42\% e $41 \%$, respectivamente). Também foi observada evolução na publicação de artigos em periódicos de circulação nacional; crescimento gradativo naqueles de circulação internacional e tendência de estagnação na publicação de livros e capítulos de livros. 0 crescimento do número de grupos de pesquisa e da produção intelectual reforçam o desenvolvimento e consolidação da Educação Física enquanto área acadêmica.

UnIteRMos: Grupos de pesquisa; Educação fisica; Indicadores de produção científica.

\section{Introdução}

A condução de pesquisas é fundamental para o desenvolvimento sociopolítico e tecnológico, constituindo-se em um componente primordial na formação acadêmico-profissional (AlgARRA, MuÑOZ \& VARGAS, 2009). Essa competência investigativa mantém interrelação com o número e qualidade de pesquisadores e de suas produçóes científicas e, especialmente, com a constituição e expansão de grupos de pesquisa. A partir da concepção de currículo enquanto construção humana (PACHECO, 1996), os grupos de pesquisa consistem em espaços paralelos de formação acadêmica na universidade, contribuindo para a realização de atividades coletivas ou compartilhadas de produção de conhecimentos (ERDMANN \& LANZONI, 2008).
A consolidação dos grupos de pesquisa no Brasil ocorreu na década de 90, principalmente em virtude do apoio de órgãos governamentais, como o Conselho Nacional de Desenvolvimento Científico e Tecnológico - CNPq (Backes, Canever, Ferraz, Lino, Prado \& Reibnitz, 2009). No caso específico da Educação Física, os primeiros grupos de pesquisa oficializados datam de 1980 e essa ascensão derivou, essencialmente, da ampliação da formação de mestres e doutores. Anteriormente a esse período, professores foram estimulados por políticas de impulsionamento acadêmico a cursar doutorado no exterior (KROEFF \& NAHAS, 2003), recebendo bolsas de estudo de órgãos de fomento, a fim de 
possibilitar efeito multiplicador na área (FARIAS JUNIOR, 1986). Tal formação de pós-graduados se deu basicamente na Alemanha e nos Estados Unidos, e o retorno de tais profissionais ao Brasil possibilitou o desenvolvimento dos primeiros cursos de Mestrado em Educação Física nos Estados do Rio Grande do Sul, São Paulo e Rio de Janeiro, dando início à "elite intelectual" responsável pela Educação Física brasileira (SILVA, 1997).

Os grupos de pesquisa vinculados aos programas de pós-graduação em Educação Física possuem ampla visibilidade e produtividade. Em função das exigências de qualidade colocadas por agências de fomento, os grupos de pesquisa consistem em importantes instrumentos capazes de gerar uma produção bastante significativa, para atender aos critérios de legitimidade acadêmica (TaVares, SchwartZ, Alves, Santiago \& KaWAGUTI, 2009). Além disso, possibilitam almejar uma progressão de conceito de avaliação e, com isso, maior reconhecimento das agências de fomento, de seus pares e da comunidade científica, como um todo.

\section{Métodos}

Trata-se de um estudo de caráter descritivo baseado em análise secundária de dados conduzida em agosto de 2010, a partir das informaçôes contidas no Diretório dos Grupos de Pesquisa no Brasil, pertencente ao Conselho Nacional de Desenvolvimento Científico e Tecnológico (CNPq, 2010).

A base de dados do Diretório é pública e contém informações sobre os grupos de pesquisa em atividade no país. Desde 1992, o CNPq realiza censos divulgando informações quantitativas sobre os grupos de pesquisa em suas diversas dimensōes. Essas informações estão disponibilizadas na página eletrônica do Diretório e podem ser acessadas por meio de diversas ferramentas, denominadas módulos. Neste estudo, o módulo "Plano Tabular" (http:// dgp.cnpq.br/planotabular/) foi utilizado. Nele, é possível o cruzamento de variáveis referentes aos grupos de pesquisa, aos seus membros (pesquisadores, estudantes e pessoal técnico), às suas linhas de pesquisa, à sua interação com o setor produtivo e à sua produção (científica, tecnológica e artística).

Os dados presentes no Diretório são coletados preferencialmente por dois meios. As informações referentes ao grupo (número de pesquisadores, estudantes, pessoal de apoio técnico e linhas de pesquisa) são adquiridas por meio de um
Ao longo dos anos, o surgimento de novos programas de pós-graduação em Educação Física e a consolidação de outros têm contribuído consideravelmente para alavancar a pesquisa na área, muito embora esse avanço ainda seja tímido, quando comparado às demais ciências tradicionalmente solidificadas e também incluídas na grande área da saúde.

É notável a carência de investigações científicas de abrangência nacional, especialmente na área da Educação Física, cuja abordagem temática relacione-se à criação e desenvolvimento de grupos de pesquisa. Tais diagnósticos servirão para identificar as peculiaridades, aspectos positivos, bem como limitaçōes com vistas ao fortalecimento da área. Sendo assim, o presente artigo buscou descrever a evolução dos grupos de pesquisa em cursos de Educação Física em geral e com programas de pós-graduação "stricto sensu”, situando-os nas macrorregiōes geográficas do Brasil, identificando o número de pesquisadores e de estudantes por grupo, além de sua produção bibliográfica no período de 2000 a 2008. questionário eletrônico padronizado, preenchido pelos líderes de cada grupo. Dados relativos à produção científica, tecnológica e artística são de responsabilidade de cada líder, pesquisador e estudante, que os informam em seus Currículos "Lattes". O cadastramento dos líderes dos grupos de pesquisa, bem como a respectiva certificação, é de responsabilidade das instituições participantes (universidades, institutos tecnológicos e empresas estatais, por exemplo) e os dados existentes nos censos se referem somente aos grupos certificados no momento do levantamento realizado pelo Diretório.

Para o estudo foram definidas como variáveis dependentes: grupos de pesquisa (conjunto de indivíduos organizados hierarquicamente, cujo fundamento organizador são a experiência, o destaque e a liderança no terreno científico ou tecnológico. Nele há envolvimento profissional e permanente com pesquisa, o trabalho é organizado em linhas de pesquisa e há o compartilhamento de instalaçôes e equipamentos); artigos nacionais (artigos publicados em revistas técnico-científicas e periódicos especializados, em língua portuguesa ou sem informação sobre o idioma); artigos internacionais (artigos publicados em outro idioma que não o português, em revistas técnico-científicas e 
periódicos especializados); livros; capítulos de livros; relação do número de pesquisadores por grupos de pesquisa; e relação do número de estudantes por grupos de pesquisa. As definições operacionais das variáveis são as mesmas adotadas pelo $\mathrm{CNPq}$ (2010) em suas coletas de informaçôes.

Como variáveis independentes foram utilizadas: macrorregiōes geográficas (Sul, Sudeste, CentroOeste, Nordeste e Norte); anos contidos no Diretório de Grupos de Pesquisa (2000, 2002, 2004, 2006 e 2008); cursos de Educação Física em geral e com programas de pós-graduação "stricto sensu", de instituiçōes brasileiras de ensino superior em cada ano pesquisado, reconhecidos pela Coordenação de Aperfeiçoamento de Pessoal de Nível Superior - CAPES (2010); cursos incluídos na grande área da saúde reconhecidos pela CAPES; número de pesquisadores; e número de estudantes.

\section{Resultados}

Os primeiros grupos de pesquisa em Educação Física no Brasil foram criados em meados da década de 80 , totalizando cinco grupos distribuídos em quatro instituições brasileiras. $\mathrm{O}$ primeiro grupo de pesquisa foi o da UFRGS/RS em 1986 e, em anos subsequentes, surgiu um grupo na PUC/SP (1987), um na USP e dois na UNICAMP (1988). Dessas instituições, apenas a $\mathrm{PUC/SP}$ não possuía programa de pós-graduação em Educação Física.

Ao considerar apenas os grupos existentes, desde sua formação até o ano de 2008, observou-se que existiam 105 grupos de pesquisa na Grande Área da Saúde até 1984. A partir de 1985, a existência de novos grupos de pesquisa tanto na Grande Área da Saúde como na Educação Física quase dobrou em relação aos períodos anteriores. Com isso, verificou-se a existência de 3961 grupos de pesquisa na Grande Área da Saúde e de 387 grupos na área de Educação Física até o ano de 2008.

\section{Evolução de grupos de pesquisa em Educação Física}

Dos grupos de pesquisa criados em Educação Física, verificou-se que até o fim do ano 2000
Embora seja incumbência dos líderes definir quem são os pesquisadores e estudantes de seu grupo, o Diretório do CNPq esclarece que devem ser considerados como pesquisadores os membros graduados ou pós-graduados da equipe de pesquisa, permanente e criativamente envolvidos com a realização de projetos e com a produção científica, tecnológica e artística do grupo. Caso esteja matriculado em curso de graduação ou pósgraduação "stricto" ou "lato sensu", o membro deve ser incluído como estudante, desde que seu orientador seja um pesquisador do grupo. Estagiários de pós-doutorado devem ser considerados como pesquisadores do grupo (CNPq, 2010).

$\mathrm{Na}$ análise dos dados foi utilizada a estatística descritiva (frequências absolutas e relativas, além de razões) conduzida no software Microsoft ${ }^{\circledR}$ Office ${ }^{\circledR}$ Excel 2007, versão para Microsoft ${ }^{\circledR}$ Windows $^{\mathrm{TM}}$.

( $\mathrm{n}=86$ grupos) a maior proporção destes estava inserida em instituições com programas de pósgraduação stricto sensu em Educação Física $(75,6 \%)$. Após este período, a quantidade de novos grupos de pesquisa aumentou $(\mathrm{n}=301$ grupos) e houve uma inversão na inserção destes, com tendência de aumento no número de grupos de pesquisa nos cursos de Educação Física em geral $(56,1 \%)$ e de estabilização naqueles com pós-graduação "stricto sensu" (43,9\%), conforme a FIGURA 1.

Ao considerar a macrorregião geográfica, observou-se no ano de 2000 que $86,7 \%$ dos grupos de pesquisa existentes nos cursos de Educação Física e 93,0\% daqueles com pós-graduação em Educação Física estavam concentrados nas regiōes sul e sudeste do país. Em 2002 observou-se um aumento na proporção de grupos de pesquisa na região sul, e nos anos subsequentes, aumento nos grupos de pesquisa nos cursos de Educação Física nas regiōes nordeste e centro-oeste (FIGURA 2). 
Del DUCA, G.F. et al.

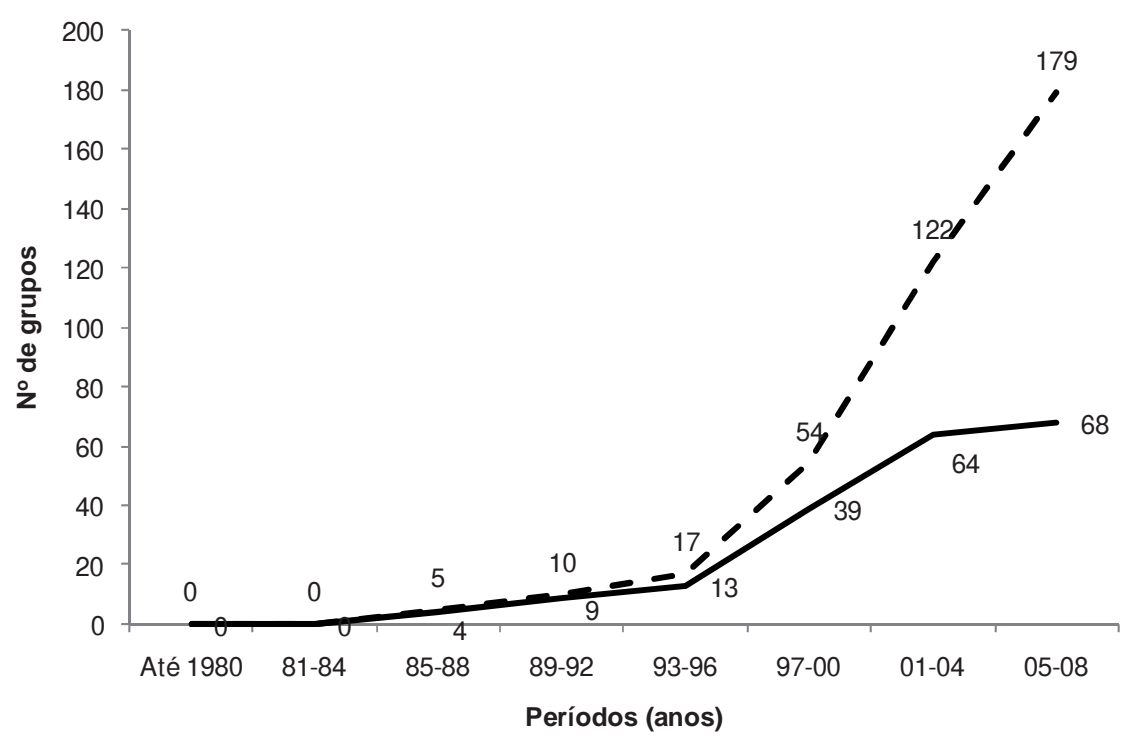

FIGURA 1 - Número de grupos de pesquisa criados em cursos de Educação Física no total e com pós-graduação "stricto sensu" ao longo dos anos.

PPGEF: Grupos de pesquisa em Programas de Pós-graduação em Educação Física;

GEF: Grupos de pesquisa em Cursos de Educação Física em geral.

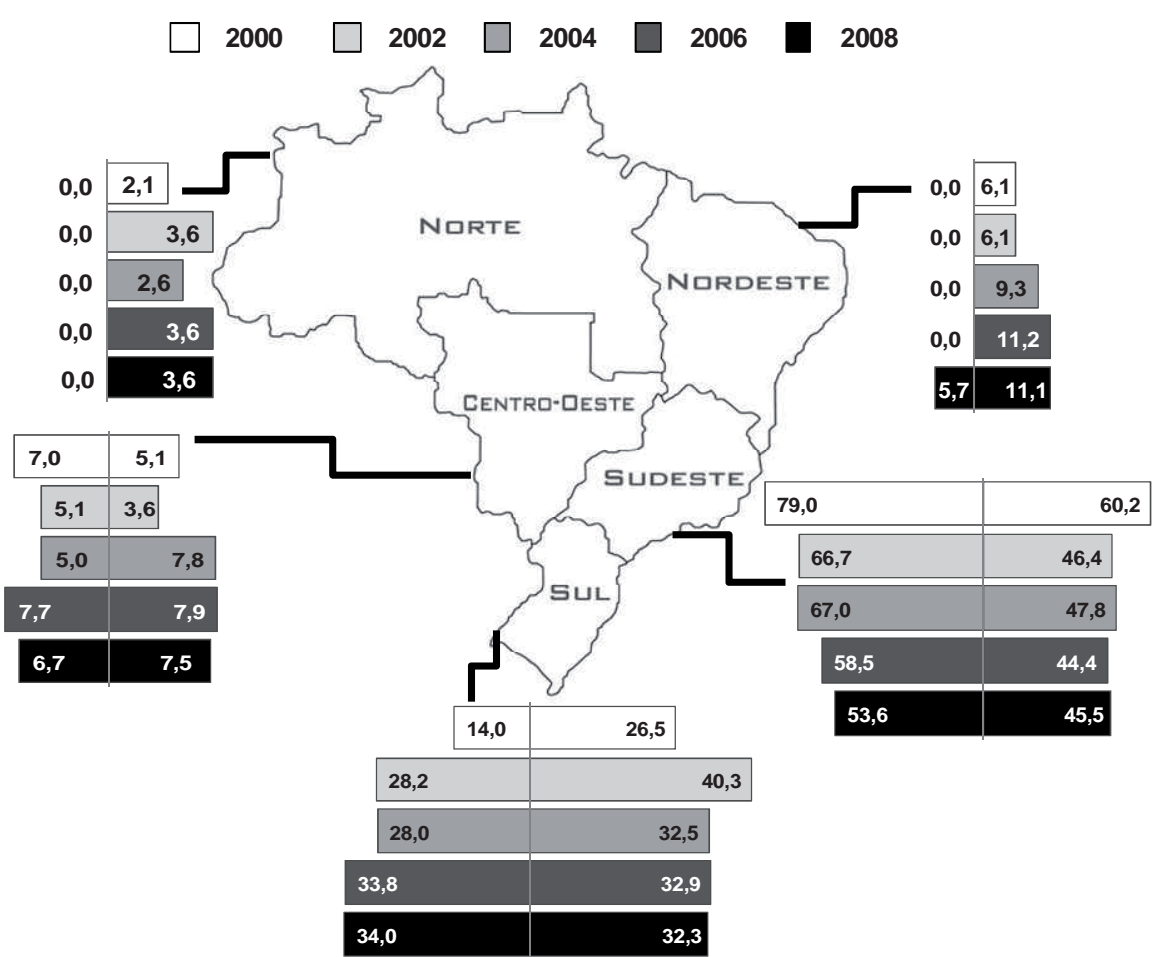

FIGURA 2 - Percentual de grupos de pesquisa nos cursos de Educação Física, em geral (barras à direita) e com pós-graduação “stricto sensu” (barras à esquerda), por macrorregião geográfica do Brasil. 


\section{Relação do número de pesquisadores e de estudantes por grupos de pesquisa em Educação Física}

No ano 2000 havia 453 pesquisadores nos cursos de Educação Física e, nos anos subsequentes, observou-se aumento substancial do número de pesquisadores, quando confrontados com o ano anterior (2002: aumento de 89\%; 2004: 71\%; 2006: 34\% e 2008: 27\%). Quanto ao número de estudantes, existiam 479 em 2000, com aumento da proporção nos anos subsequentes em comparação ao ano anterior (2002: aumento de 83\%; 2004: 76\%; 2006: 42\% e 2008: 41\%).

A relação do número de pesquisadores por grupo de pesquisa aumentou ao longo dos anos (2000: 4,6; 2002: 4,4; 2004: 5,5; 2006: 6,5 e 2008: 6,5), com destaque para a região sudeste (2000: 2,6; 2002: 4,0; 2004: 5,3; 2006: 6,8 e 2008: 6,4) e tendência de estabilidade na região sul (2000: 5,9; 2002: 4,8; 2004: 5,9; 2006: 6,2 e 2008: 6,5). As demais macrorregiōes geográficas apresentaram oscilações nos valores ao longo dos anos, observando equilíbrio do número de pesquisadores / grupo (de 6 a 7 ) entre as regióes no ano de 2008.

O número de estudantes por grupo também evoluiu (2000: 4,9; 2002: 4,5; 2004: 5,8; 2006: 7,2 e 2008: $8,0)$, revelando uma crescente participação dos alunos nos grupos de pesquisa na macrorregião Sul do país (2000: 3,0; 2002: 3,9; 2004: 6,2; 2006: 7,8 e 2008: $9,2)$, com estabilidade e picos de crescimento nas regiōes
Nordeste (2000: 5,3; 2002: 2,0; 2004: 5,4; 2006: 7,6 e 2008: 8,4) e Sudeste (2000: 5,7; 2002: 4,8; 2004: 6,0; 2006: 7,4 e 2008: 7,4), e instabilidade na macrorregiāo Centro-Oeste (2000: 4,8; 2002: 7,9; 2004: 4,0; 2006 : 5,0 e 2008: 6,5) e Norte (2000: 4,0; 2002: 4,8; 2004 : 2,6; 2006: 3,5 e 2008: 7,3) do Brasil.

\section{Produção bibliográfica de pesquisadores e de estudantes envolvidos na pós-graduação em Educação Física}

No ano 2000 foram publicados 137 artigos em periódicos de circulação nacional e, em anos subsequentes, observou-se um aumentou considerável (2002: 403\%; 2004: 70\%; 2006: 99\% e 2008: 26\%) neste tipo de publicação, ao comparar com o ano anterior. Foram computados 38 artigos em periódicos de circulação internacional no ano 2000 e também foi observado que a proporção de artigos desta natureza aumentou nos anos subsequentes (2002: 242\%; 2004: 290\%; 2006: 204\% e 2008: 45\%), em relação aos anteriores.

A produção de livros $(\mathrm{n}=9)$ e de capítulos de livros $(\mathrm{n}=44)$ no ano 2000 aumentou em cinco vezes no ano de 2002 ( $\mathrm{n}=49$ e $\mathrm{n}=248$, respectivamente). Nos anos subsequentes, o aumento ocorreu em menor proporção, tanto para os livros (2004: 67\% e 2006: 38\%) quanto para os capítulos de livros (2004: 73\% e 2006: 40\%), com apenas $5 \%$ de aumento em capítulos de livros e declínio de 14\% na produção de livros em 2008 (FIGURA 3).

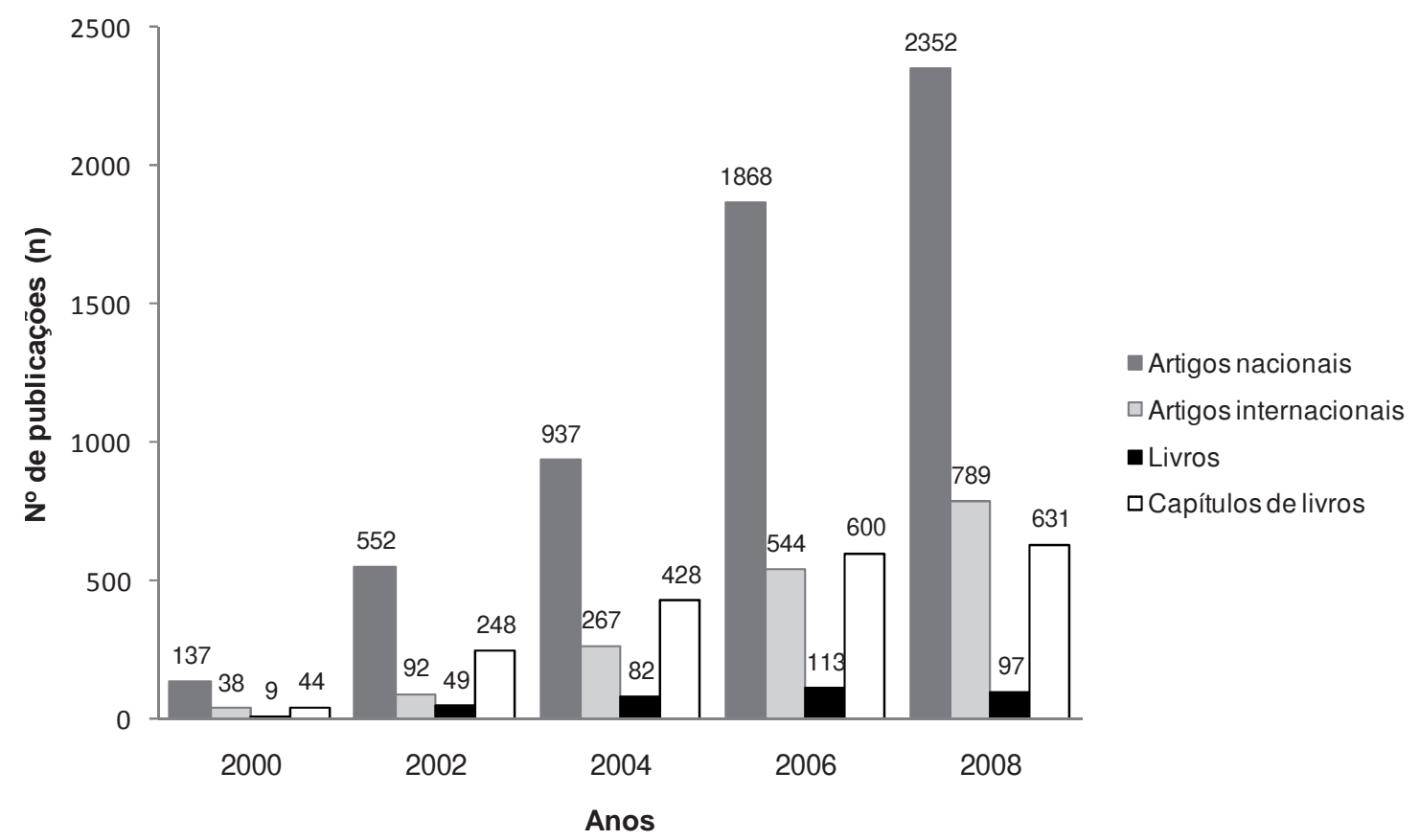

FIGURA 3 - Número de publicações dos grupos de pesquisa em cursos de Educação Física com pós-graduação "stricto sensu". 
Na TABELA 1, observou-se que quatro instituições possuíam e mantiveram um programa com mestrado/ doutorado de 2000 a 2008 (IES 9, 14, 17 e 23) e seis obtiveram credenciamento para o mestrado/ doutorado em anos subsequentes (IES 1, 3, 6, 8, 11 e 16). O número de artigos de circulação nacional por pesquisadores envolvidos em grupos de pesquisa de universidades com programa de pós-graduação em Educação Física foi inferior a um, no ano 2000, com evolução ao longo dos anos. A produção de artigos de circulação internacional por pesquisadores pertencentes a grupos de pesquisa de universidades com programa de pós-graduação em Educação Física foi inferior a um em 14 instituições das 23 analisadas.

TABELA 1 - Produção deartigos nacionais e internacionais no período de 2000 a 2008, deacordo com o número de pesquisadores em grupos de pesquisa de instituições com pós-graduação "stricto sensu" em Educação Física.

\begin{tabular}{|c|c|c|c|c|c|c|c|c|c|c|c|}
\hline \multirow{25}{*}{$\begin{array}{l}\text { (-) Instituições que não } \\
\text { possuíam mestrado; } \\
\text { (valores sem negrito) } \\
\text { Instituições somente } \\
\text { com mestrado; } \\
\text { (valores com negrito) } \\
\text { Instituições com dou- } \\
\text { torado. }\end{array}$} & \multirow{2}{*}{ IES } & \multicolumn{5}{|c|}{ Artigos nacionais } & \multicolumn{5}{|c|}{ Artigos internacionais } \\
\hline & & 00 & 02 & 04 & 06 & 08 & 00 & 02 & 04 & 06 & 08 \\
\hline & 1 & 0,5 & 1,5 & 0,8 & 1,8 & 1,7 & 0,1 & 0,6 & 0,5 & 0,8 & 1,1 \\
\hline & 2 & 0,4 & 1,2 & 1,4 & 1,2 & - & 0,1 & 0,1 & 0,2 & 0,1 & - \\
\hline & 3 & 0,0 & 0,7 & 0,9 & 1,4 & 1,6 & 0,1 & 0,1 & 0,1 & 0,6 & 0,3 \\
\hline & 4 & - & - & - & 1,8 & 1,3 & - & - & - & 0,3 & 1,4 \\
\hline & 5 & - & - & - & 1,1 & 1,1 & - & - & - & 0,3 & 0,1 \\
\hline & 6 & 0,3 & 1,9 & 1,5 & 1,7 & 0,6 & 0,0 & 1,3 & 1,0 & 0,8 & 0,4 \\
\hline & 7 & - & - & - & - & 1,4 & - & - & - & - & 0,6 \\
\hline & 8 & - & 0,7 & 1,0 & 1,4 & 1,5 & - & 0,2 & 1,0 & 0,5 & 0,4 \\
\hline & 9 & 0,8 & 2,1 & 0,8 & 1,1 & 1,4 & 0,0 & 0,3 & 0,2 & 0,5 & 0,5 \\
\hline & 10 & - & - & - & - & 3,4 & - & - & - & - & 0,7 \\
\hline & 11 & 0,5 & 1,1 & 0,7 & 1,1 & 2,1 & 0,0 & 0,0 & 0,1 & 0,3 & 1,0 \\
\hline & 12 & 0,4 & - & - & - & - & 0,0 & - & - & - & - \\
\hline & 13 & - & - & - & - & 1,9 & - & - & - & - & 0,3 \\
\hline & 14 & 0,0 & 15,0 & 1,3 & 0,6 & 0,7 & 0,0 & 1,5 & 0,5 & 0,4 & 0,3 \\
\hline & 15 & - & - & - & 0,5 & 0,4 & - & - & - & 0,1 & 0,1 \\
\hline & 16 & 0,4 & 1,6 & 1,5 & 1,7 & 1,6 & 0,1 & 0,2 & 0,8 & 0,5 & 1,0 \\
\hline & 17 & 0,4 & 0,9 & 1,6 & 1,8 & 1,2 & 0,2 & 0,0 & 0,4 & 0,5 & 0,2 \\
\hline & 18 & - & - & - & - & 0,5 & - & - & - & - & 3,1 \\
\hline & 19 & 0,2 & 0,8 & 0,4 & 1,2 & 1,2 & 0,2 & 0,2 & 0,2 & 0,1 & 0,2 \\
\hline & 20 & - & - & - & 1,0 & 1,5 & - & - & - & 0,3 & 0,3 \\
\hline & 21 & - & - & - & - & 0,9 & - & - & - & - & 0,1 \\
\hline & 22 & - & - & 1,3 & 1,0 & 1,4 & - & - & 0,5 & 1,0 & 0,6 \\
\hline & 23 & 0,7 & 1,1 & 5,8 & 1,6 & 2,0 & 0,3 & 0,5 & 1,7 & 0,9 & 1,1 \\
\hline
\end{tabular}

$\mathrm{Na}$ análise envolvendo publicação de artigos por número de estudantes envolvidos em instituições com programa de pós-graduação em Educação Física (TABELA 2) verificou-se que quatro instituições (IES 3, 8, 11 e 19) apresentaram, pelo menos, um artigo por estudante em três ou mais anos. Nenhuma instituição investigada atingiu o valor de um artigo por estudante em publicação de circulação internacional.
Os dados demonstraram uma relação inferior a um na produção de livros por pesquisador, não ultrapassando 0,2 no ano de 2008. Para os capítulos de livros verificou-se que a relação foi de um ou mais em algumas instituições nos quatro primeiros anos. Entretanto, no último ano somente a IES 10 apresentou uma relação de um capítulo de livro por pesquisador. A relação de produção de livros no corpo discente destas instituiçôes foi de 0,3 e a de capítulos de livros chegou a 0,7 apenas na IES 16. 
TABELA 2 - Produção de artigos nacionais e internacionais no período de 2000 a 2008, de acordo com o número de estudantes em grupos de pesquisa de instituições com pós-graduação "stricto sensu" em Educação Física.

\begin{tabular}{|c|c|c|c|c|c|c|c|c|c|c|}
\hline \multirow{2}{*}{ IES } & \multicolumn{5}{|c|}{ Artigos nacionais } & \multicolumn{5}{|c|}{ Artigos internacionais } \\
\hline & 00 & 02 & 04 & 06 & 08 & 00 & 02 & 04 & 06 & 08 \\
\hline 1 & 0,0 & 0,7 & 0,5 & 0,9 & 1,5 & 0,0 & 0,0 & 0,1 & 0,2 & 0,8 \\
\hline 2 & 0,0 & 0,3 & 0,5 & 0,5 & - & 0,0 & 0,0 & 0,1 & 0,1 & - \\
\hline 3 & 0,0 & 0,2 & 2,0 & 1,4 & 1,7 & 0,0 & 0,0 & 0,1 & 0,6 & 0,4 \\
\hline 4 & - & - & - & 1,5 & 1,4 & - & - & - & 0,1 & 0,3 \\
\hline 5 & - & - & - & 0,1 & 0,3 & - & - & - & 0,1 & 0,0 \\
\hline 6 & 0,0 & 0,1 & 1,0 & 0,8 & 0,2 & 0,0 & 0,0 & 0,8 & 0,6 & 0,0 \\
\hline 7 & - & - & - & - & 0,3 & - & - & - & - & 0,0 \\
\hline 8 & - & 1,0 & 1,4 & 1,5 & 1,0 & - & 0,0 & 0,4 & 0,7 & 0,2 \\
\hline 9 & 0,5 & 0,7 & 0,6 & 1,0 & 1,8 & 0,0 & 0,1 & 0,1 & 0,3 & 0,6 \\
\hline 10 & - & - & - & - & 0,6 & - & - & - & - & 0,1 \\
\hline 11 & 0,8 & 0,5 & 1,5 & 2,4 & 3,4 & 0,0 & 0,1 & 0,3 & 0,2 & 0,2 \\
\hline 12 & 0,0 & - & - & - & - & 0,0 & - & - & - & - \\
\hline 13 & - & - & - & - & 1,0 & - & - & - & - & 0,2 \\
\hline 14 & 0,0 & 1,2 & 0,3 & 0,8 & 0,5 & 0,0 & 0,0 & 0,0 & 0,3 & 0,3 \\
\hline 15 & - & - & - & 0,5 & 0,3 & - & - & - & 0,2 & 0,0 \\
\hline 16 & 0,1 & 0,7 & 0,7 & 1,7 & 1,6 & 0,1 & 0,0 & 0,2 & 0,3 & 0,3 \\
\hline 17 & 0,1 & 0,3 & 0,7 & 1,0 & 0,7 & 0,0 & 0,0 & 0,0 & 0,1 & 0,1 \\
\hline 18 & - & - & - & - & 0,0 & - & - & - & - & 0,0 \\
\hline 19 & 0,0 & 1,0 & 0,3 & 1,9 & 1,2 & 0,0 & 0,0 & 0,1 & 0,0 & 0,2 \\
\hline 20 & - & - & - & 0,5 & 0,3 & - & - & - & 0,0 & 0,0 \\
\hline 21 & - & - & - & - & 0,3 & - & - & - & - & 0,0 \\
\hline 22 & - & - & 0,4 & 0,6 & 0,5 & - & - & 0,0 & 0,5 & 0,3 \\
\hline 23 & 0,1 & 0,2 & 0,4 & 0,8 & 0,8 & 0,0 & 0,0 & 0,1 & 0,2 & 0,4 \\
\hline
\end{tabular}

(-) Instituições que não possuíam mestrado; (valores sem negrito) Instituições somente com mestrado;

(valores com negrito) Instituições com doutorado.

\section{Discussão}

O considerável aumento do número de pesquisadores em Educação Física e da representatividade destes, em termos percentuais, na Grande Área da Saúde é reflexo inicial das políticas de incentivo à formação de mestres e doutores em Educação Física implementadas na década de 70 (Kroeff \& Nahas, 2003). O número crescente de pesquisadores repercute, ao mesmo tempo, em maior desenvolvimento da produção científica e maior concorrência entre os pesquisadores (Mocelin, 2009). Um aspecto a ser destacado é que a expansão pode ser explicada não apenas em função do reconhecimento da importância do conhecimento científico, mas da valorização econômica atrelada à pesquisa. Esse fato pode ser explicado pelo crescente apoio financeiro das agências de fomento nacionais e internacionais que, a partir de editais, custeiam pesquisas e recursos humanos, conforme critérios pré-estabelecidos, como por exemplo, relevância do estudo e regularidade e qualidade da produção científica do pesquisador. De fato, essa política possibilitou tanto a maior concorrência quanto a formação de novas alianças entre pesquisadores, em busca de recursos financeiros, conhecimento acadêmico e credibilidade. Além disso, favoreceu o desenvolvimento do trabalho realizado em conjunto, como no caso dos grupos de pesquisa.

Observaram-se neste estudo dois tipos de tendência quanto à formação de novos grupos de pesquisa em Educação Física. A primeira delas reside na 
estabilização do número de grupos oriundos de cursos com pós-graduação "stricto sensu"; já a segunda e animadora observação diz respeito ao crescente aumento de grupos de pesquisa em cursos de Educação Física na sua totalidade, o que evidencia a preocupação da grande maioria das instituiçōes de ensino superior (que não possuem cursos de mestrado e doutorado em seus currículos), bem como de profissionais, estudantes e técnicos que reconhecem e se utilizam da estratégia de grupos de pesquisa para aprimorar a formação acadêmica. Entretanto, é importante destacar que o crescimento do número de programas de pós-graduação "stricto sensu" não acompanha o aumento exponencial dos cursos de graduação em Educação Física (SANTOS \& SimŌES, 2008). Esse fato pode ser explicado tanto pela política de expansão dos cursos de graduação, quanto pelo aumento do rigor do processo de credenciamento e recredenciamento dos cursos de pós-graduação. A ampliação do número de cursos de nível superior, ocorrida a partir da Lei de Diretrizes e Bases da Educação Nacional (BRAsiL, 1996), teve como princípio o aumento do nível de escolaridade da população brasileira, com vistas a reduzir as desigualdades socais. Em contrapartida, a criação de novos programas de pós-graduação em Educação Física depende do atendimento de critérios estabelecidos pelo Ministério da Educação, bem como pela disponibilidade de recursos financeiros para atender às demandas específicas que esta formação requer.

A maior proporção de grupos de pesquisa em Educação Física está localizada nas macrorregiōes sudeste e sul do país. Além dos indicadores econômicos e da elevada concentração de cursos de graduação nessas regiōes, esta situação parece refletir a própria história e evolução da Educação Física no Brasil. Foi exatamente nestes dois cenários que surgiram os primeiros grupos de pesquisa em Educação Física e os primeiros programas de pós-graduação, os quais contaram com a inserção dos profissionais pósgraduados que, naquela época, retornavam da Europa ou dos Estados Unidos. Entretanto, nos últimos três anos investigados observou-se tendência de estabilidade nessas macrorregiōes e, a partir de 2004, uma evolução importante na macrorregião nordeste e no centro-oeste. Somente a região norte do país tem apresentado, ainda, um discreto percentual de aumento dos grupos de pesquisa.

Apesar de existir políticas nas agências de fomento para minimizar as distorções regionais e favorecer a criação de programas de pós-graduação em Educação
Física na região Centro-Oeste, Nordeste e Norte do Brasil, tem-se observado que as disparidades regionais de oferta nesta modalidade continuam, visto que das 22 instituiçôes brasileiras com programas de pós-graduação em Educação Física, somente duas estão na macrorregião do Centro-Oeste e uma na Nordeste, o que influencia diretamente o número de grupos de pesquisa neste sistema.

A abertura de programas de pós-graduação "stricto sensu" está condicionada ao cumprimento de critérios estabelecidos pela CAPES, bem como a sua manutenção está atrelada ao conceito obtido no processo de avaliação trienal. Dentre os critérios exigidos, destaca-se a regularidade e qualidade da produção intelectual que engloba a publicação de artigos científicos em periódicos de circulação nacional e internacional, a publicação de livros e de capítulos de livros (COSTA, 2008). Cabe ressaltar que a estratificação da qualidade da produção intelectual adotada pela CAPES visa somente à avaliação dos programas de pós-graduação e não se destina à avaliação da produção individual de professores, pesquisadores e estudantes. De fato, há preocupação nas instituiçôes brasileiras em atingir os critérios quantitativos e qualitativos de produção intelectual como meio de obter grau de excelência e modernidade, havendo um incentivo hegemônico para a publicação de artigos (Carvalho \& Manoel, 2007).

Quanto à queda da produção de livros, duas correntes de ideias principais têm sido discutidas. A primeira sugere que recentemente ocorreu uma valorização exacerbada da produção de artigos científicos e, ao mesmo tempo, uma desvalorização do livro na avaliação dos programas de pós-graduação. Por conseguinte, os artigos foram utilizados como forma preferencial de compartilhamento de pesquisas e conhecimentos na área. A segunda corrente propõe que, na verdade, não houve uma redução do número de livros publicados nos últimos anos, mas sim dos livros que atendiam aos critérios de avaliação da CAPES, mais rigorosos quanto à qualidade do material publicado. Ou seja, uma parcela dos livros publicados pela área não atingiram os níveis adequados de qualidade e por isso não foram considerados como produção técnica.

As evidências encontradas parecem acompanhar esta situação, considerando que foi observada uma acentuada evolução nas publicações de artigos em periódicos de circulação nacional no ano de 2002 e em proporção menor nos demais anos, com crescimento gradativo naqueles de circulação internacional e estagnação, com tendência à redução de publicação 
de livros e capítulos de livros, tanto por pesquisadores quanto por estudantes, até o ano de 2008.

A valorização da área acadêmica, enquanto campo de intervenção do futuro profissional, tem provocado um aumento considerável na procura por uma formação acadêmica de melhor qualidade em Educação Física, e a pesquisa é parte integral do exercício profissional, devendo estar incluída na formação de mestres e doutores (AlgarRa, MuÑoz \& VARGAS, 2009). Tal fato justifica o importante crescimento quanti-qualitativo das publicaçôes produzidas em Educação Física, bem como a melhor qualificação de graduandos, mestres e doutores da área e no fortalecimento dos grupos de pesquisa. $\mathrm{Na}$ atualidade, os estudantes possuem maiores oportunidades de ingressar em grupos de pesquisa, ainda na formação inicial. Além de ter novas possibilidades de aprendizagem teórico-práticas, há também o aprimoramento técnico-científico que enriquece a formação inicial, que pode, sem dúvida, estimulá-lo à formação continuada.

A evidência de que cursos que possibilitam a formação de doutores também alcançam valores mais elevados de produção de artigos, tanto por pesquisadores quanto por estudantes em seus grupos de pesquisas, quando comparados aos demais, reforçam dois aspectos. Primeiramente, que o crescimento e o fortalecimento da pós-graduação em Educação Física no Brasil passam, necessariamente, pela pesquisa e produção de conhecimentos, articuladas principalmente nos grupos de pesquisa. Em outras palavras, a formação de mestres e doutores é altamente dependente do grau de consolidação da pesquisa (KoKUBUn, 2003), e esta do trabalho que ocorre nos grupos de pesquisa. Por outro lado, a formação de pesquisadores qualificados para tais tarefas depende da capacidade instalada e dos recursos humanos do sistema de pós-graduação (Costa \& NAscimento, 2008). Trata-se, portanto, de um ciclo virtuoso envolvendo ensino e pesquisa, em que os grupos de pesquisa desempenham papel fundamental.

Quanto aos aspectos positivos deste artigo, não é do nosso conhecimento a publicação de outro trabalho que aborde os grupos de pesquisa em Educação Física com abordagem similar. Além disso, a utilização da análise temporal permitiu registrar a evolução dos fenômenos e identificar tendências. Por último, por conta da abrangência do banco de dados, foi possível fazer uma análise nacional e observar características e disparidades entre as macrorregiōes geográficas.

Entretanto, alguns pontos merecem atenção na interpretação dos resultados. Primeiro, a existência de dupla ou múltipla contagem em algumas informações. Os pesquisadores e estudantes que participam em mais de um grupo são contabilizados em cada grupo, assim como suas publicaçôes. Da mesma forma, produções envolvendo membros de um mesmo grupo são contabilizadas uma vez para cada membro autor. Segundo, podem fazer parte dos grupos de pesquisa em Educação Física membros envolvidos com pesquisa e produção de conhecimento em outras áreas. Nestes casos, sua produção nas demais áreas também foi contabilizada. Além disso, por se tratar de uma base constituída por dados fornecidos, em parte, por pesquisadores líderes, provavelmente houve casos de não inclusão de grupos de pesquisa na presente análise, em função da desatualização de informaçōes.

\section{Conclusão}

O aumento considerável na série histórica de 2000 a 2008 do número de grupos de pesquisa, da relação pesquisador e de estudante por grupo e, consequentemente, da produção intelectual, em grande parte resulta das publicaçōes de artigos científicos, reforçam o desenvolvimento e a consolidação da Educação Física enquanto área acadêmica. Contudo, observaram-se grandes disparidades regionais, assim como a menor participação dos livros como forma de construção do conhecimento.

$\mathrm{O}$ incremento de políticas nacionais, como a destinação de parte dos recursos de pesquisa para fomentar grupos emergentes nas regiōes Norte e Nordeste, assim como incentivar à abertura de programas de pós-graduação "stricto sensu" são necessários para minimizar as desigualdades regionais. Acredita-se que as recentes mudanças no sistema de avaliação da produção intelectual implementadas na CAPES, que contemplam a avaliação dos livros como forma de produção do conhecimento, resgate sua devida importância.

As investigaçōes futuras nesta área necessitam abordar os modelos de ramificação adotados pelos grupos de pesquisa e as respectivas temáticas 
investigativas. Além disso, há necessidade de comparações mais ampliadas do curso de Educação Física com os demais cursos da grande área da saúde, assim como contemplação das principais estratégias adotadas para o desenvolvimento científico, as normas reguladoras e as especificidades do trabalho científico.

\begin{abstract}
Research groups in Physical Education graduate programs in Brazil: trend analysis from 2000 to 2008

The aim of this study was to describe the evolution of research groups in Physical Education graduate programs in Brazil and their respective scientific production in the period from 2000 to 2008. This was a descriptive study, including secondary data analysis conducted in 2010 with information from the Directory of Research Groups in Brazil of the National Council for Scientific and Technological Development (CNPq). We analyzed the growth of the research groups, researchers and students to research groups ratio, national and international manuscripts production, books, and book chapters, according to geographical regions, years of data collection, and number of courses in Physical Education, researchers and students. We used descriptive statistics (ratios, absolute and relative frequencies) for analysis. The first research group in Physical Education in Brazil was created in the 80's, and in 2008 totalized 387. There was a decrease of the representativeness of groups created in institutions with graduate programs (up to 2000: 75.6\% 2005-2008: 43.9\%). In 2000, there were 453 researchers and 479 students in physical education courses. We identified an increase in the years 2002, 2004, 2006 and 2008, compared to their previous years (researchers: 89\%, 71\%, 34\% and 27\% increase; students: 83\%, 76\%, 42\% and 41 increase, respectively). Evolution was also observed in the publication of papers of national circulation; gradual increase in those with international circulation and no significant change in book and book chapters publications. The growing number of research groups and scientific publications parallels the development and consolidation of Physical Education as an academic area.
\end{abstract}

UnITERMS: Physical education; Scientific publication indicators; Research groups.

\title{
Referências
}

ALGARRA, A.J.C.; MUÑOZ, L.; VARGAS, M.P. Creación y evolución del grupo de investigación: perspectivas de cuidado definición de las líneas de investigación. Repertorio de Medicina y Cirugía, Bogotá, v.18, n.3, p.161-5, 2009. BACKES, V.M.S.; CANEVER, B.P.; FERRAZ, F.; LINO, M.M.; PRADO, M.L.; REIBNITZ, K.S. Grupos de pesquisa de educação em enfermagem da região sul do Brasil. Revista Gaúcha de Enfermagem, Porto Alegre, v.30, n.2, p.249-56, 2009. BRASIL. Lei no 9.394, de 20 de dezembro de 1996. Lei de Diretrizes e Bases da Educação Nacional. Brasília: MEC, 1996. CARVALHO, Y.M.; MANOEL, E.J. O livro como indicador da produção intelectual na grande área da Saúde. Revista Brasileira de Ciências do Esporte, Campinas, v.29, n.1, p.61-73, 2007. CONSELHO NACIONAL DE DESENVOLVIMENTO CIENTÍFICO E TECNOLÓGICO (CNPq). Diretório dos grupos de pesquisa no Brasil. Acesso em: 05/10/2010. Disponível em: http://gp.cnpq.br/censos/perguntas/perguntas.htm. COORDENAÇÃO DE APERFEIÇOAMENTO DE PESSOAL DE NÍVEL SUPERIOR (CAPES). Resultado da avaliação de programas. Disponível em: <http://www.capes.gov.br/avaliacao/resultados-da-avaliacao-de-programas > Acesso em: 01 ago. 2010.

COSTA, D.; NASCIMENTO, J.V. Mudanças no sistema de avaliação dos programas de pós-graduação. Revista Brasileira de Fisioterapia, São Carlos, v.12, n.4, p.v-vi, 2008.

ERDMANN, A.L.; LANZONI, G.M.M. Características dos grupos de pesquisa da enfermagem brasileira certificados pelo CNPq de 2005 a 2007. Escola Anna Nery: Revista de Enfermagem, Rio de Janeiro, v.12, n.2, p.316-22, 2008.

FARIA JUNIOR, A.G. Produção científica brasileira em educação física: dissertação de mestrado. Niterói: Universidade Federal Fluminense / Universidade Técnica de Lisboa, 1986. 
KOKUBUN E. Pós-graduação em educação física no Brasil: indicadores objetivos dos desafios e das perspectivas. Revista Brasileira de Ciências do Esporte, Campinas, v.24,n.2, p.9-26, 2003.

KROEFF, M.S.; NAHAS, M.V. Ações governamentais e formação de pesquisadores em Educação Física no Brasil. Revista Brasileira de Ciências do Esporte, Campinas, v.24, n.2, p.114-26, 2003.

MOCELIN, D.G. Concorrência e alianças entre pesquisadores: reflexôes acerca da expansão de grupos de pesquisa dos anos 1990 aos 2000 no Brasil. Revista Brasileira de Pós-Graduação, Brasília, v.6, n.11, p.35-64, 2009.

PACHECO, J.A. Currículo: teoria e práxis. Porto: Porto Editora, 1996.

SANTOS, A.L.P.; SIMŌES, A.C. Desafios do ensino superior em educação física: considerações sobre a política de avaliação de cursos. Ensaio: Avaliação e Políticas Públicas em Educação, Rio de Janeiro, v.16, n.59, p.259-74, 2008.

SILVA, R.V.S. Pesquisa em educação física: determinaçōes históricas e implicações epistemológicas. 1997. 279 f. Tese (Doutorado) - Universidade de Campinas, Campinas, 1997.

TAVARES, G.H.; SCHWARTZ, G.M.; ALVES, H.B.; SANTIAGO, D.R.; KAWAGUTI, C.N. Gestão do lazer: os grupos de pesquisa em foco. Motriz, Rio Claro, v.15, n.3, p.470-80, 2009.

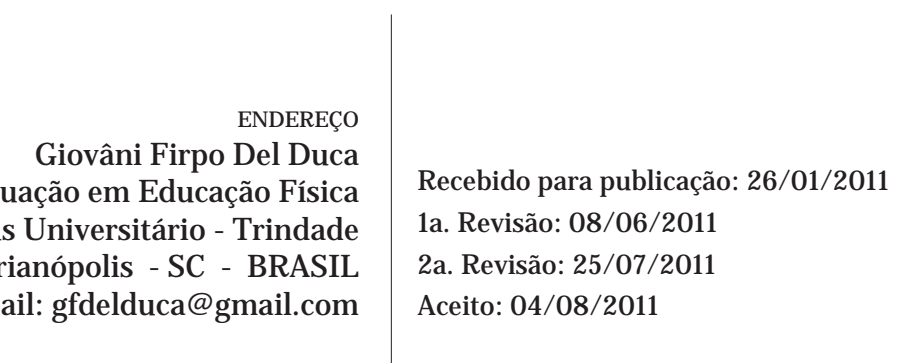

